\title{
Efecto del cadmio sobre la mortalidad de adultos de Polydora sp. (Polychaeta: Spionidae) en el laboratorio
}

\section{Melissa Herrera-Perez ${ }^{1,2}$ \& Nuria Méndez ${ }^{3}$}

1. Laboratorio de Ecotoxicología Acuática. Área Funcional de Investigaciones Marino Costeras (AFIMC) Dirección General de Investigaciones en Acuicultura (DGIA). Instituto del Mar del Perú (IMARPE). Callao, Perú; imelissa.herrera.p@gmail.com

2. Facultad de Ciencias Biológicas. Universidad Ricardo Palma (URP). Santiago de Surco, Lima, Perú.

3. Unidad Académica Mazatlán. Instituto de Ciencias del Mar y Limnología, Universidad Nacional Autónoma de México. Apartado Postal 811, Mazatlán 82000, Sinaloa, México; nuri@ola.icmyl.unam.mx

$$
\text { Recibido 03-XII-2018. Corregido 25-V-2019. Aceptado 30-VI-2019. }
$$

\begin{abstract}
Effect of cadmium on adult mortality of Polydora sp. (Polychaeta: Spionidae) under laboratory conditions. Introduction: Several studies have demonstrated the effect of $\mathrm{Cd}$ on mortality and other physiological responses of some polychaete species. Objective: to evaluate the toxic effect of Cd on adults mortality of Polydora sp as a test species for ecotoxicological studies. Methods: The specimens were collected in the coastal wetland of Poza de la Arenilla, Callao, Peru. The organisms were acclimatized for two weeks with seawater and sediment and then, three days in seawater, with $30 \%$ of salinity and temperature between 20 to $22{ }^{\circ} \mathrm{C}$. The organisms were exposed to the nominal concentrations $75,11.25,1.69,0.25 ; 0.04 \mathrm{mg} / \mathrm{L}$ of Cd and a control. Three replicates of ten individuals per treatment were analyzed for 72 hours to register mortality. The Kruskal-Wallis and U-Man Whitney tests were applied to detect significant differences among treatments. Results: The adverse effect of $\mathrm{Cd}$ on mortality was much more evident in the treatments of $11.25 \mathrm{and} 75 \mathrm{mg} / \mathrm{L}$ after the first 24 hours of exposure. The mean lethal concentration of $\mathrm{Cd}$ at $72 \mathrm{~h}$ was $2.59 \mathrm{mg} / \mathrm{L}$. Conclusions: the $\mathrm{LC}_{50}$ is in the same order of magnitude as other polychaete species. This is the first ecotoxicological study in Peru using polychaetes under laboratory conditions. It is suggested the use of Polydora sp as a test species in ecotoxicology experiments, using nominal sublethal concentrations of $\mathrm{Cd}$ not exceeding $1.6 \mathrm{mg} / \mathrm{L}$. for the observation of several physiological responses.
\end{abstract}

Key words: Ecotoxicology, polychaetes, heavy metal, $\mathrm{LC}_{50}$, bioassays, test species, coastal wetland Poza de la Arenilla.

Herrera-Perez, M., \& Méndez, N. (2019). Efecto del cadmio sobre la mortalidad de adultos de Polydora sp. (Polychaeta: Spionidae) en el laboratorio. Revista de Biología Tropical, 67(S5) Suplemento, S110-S118.

La Poza La Arenilla (Malecón Pardo, distrito de La Punta, en la Provincia Constitucional del Callao, Perú) se caracteriza por ser un ecosistema marino artificial creado con dos rompeolas, lo que ocasiona que tenga escasa circulación del agua. Los dos canales de entrada y salida del agua interactúan con el agua de la bahía del Callao. En estas aguas desembocan los ríos Rímac y Chillón, así como efluentes domésticos e industriales, los cuales pueden acarrear diferentes residuos tóxicos o peligrosos (Sánchez, Orozco, \& Guzmán, 2002; Correa, Tam, Pasapera, Saavedra, \& Ingunza, 2008). Entre los residuos tóxicos depositados en ambientes acuáticos destacan los hidrocarburos de petróleo y metales tóxicos 
como cadmio, cobre, plomo y arsénico, como consecuencia de actividades antropogénicas (desarrollo industrial, agrícola y/o urbano) o de forma natural (lluvias, escorrentía de los ríos, erosión, etc.) (Fu-hong \& Qi-xing, 2008) acumulándose en los sedimentos costeros, donde pueden alcanzar altas concentraciones (Ankley, Thomas, DiToro, Hansen, Mahony, Berry, Swartz, Hoke, Garrison, Allen, \& Zarba, 1994). En estas áreas, los organismos bentónicos pueden acumular $\mathrm{Cd}$ a través del agua y, sobre todo, de partículas ingeridas (Selck, Forbes, \& Forbes, 1998). Los niveles de Cd en el agua en ambientes marinos pueden fluctuar entre $0.005 \mathrm{ug} \mathrm{L}^{-1}$ (Nürnberg, 1977) y $130 \mathrm{ug}$ de $\mathrm{L}^{-1}$ (Förstner \& Wittmann, 1981). El Cd se encuentra como $\mathrm{CdCl}_{2}$, siendo la salinidad un factor importante que puede modificar la actividad del ion libre del $\mathrm{Cd}(+2) \mathrm{y}$, por consiguiente, la biodisponibilidad, el metabolismo y toxicidad en los sistemas marinos (Jenkins \& Sanders, 1986).

Los poliquetos (Clase Polychaeta, Phylum Annelida), son excelentes indicadores de perturbación ambiental (Tovar-Hernández, Salazar-Silva, de León-González, Carrera-Parra, \& Salazar-Vallejo, 2014; Fernández-Rodríguez \& Londoño-Mesa, 2015). Destaca la familia Spionidae (Grube, 1850) por su alta diversidad, por presentar un mayor número de especies descritas y por presentar una amplia distribución en sistemas bentónicos (Tomassetti \& Porrello, 2005). Los miembros de Polydora (Bosc, 1802), son sedimentívoros (Blake \& Woodwick, 1971; Fauchald \& Jumars, 1979), y son considerados como indicadores de contaminación por materia orgánica (Dean, 2008) y metales traza, como el cadmio e hidrocarburos (Ajao \& Fagade, 1990). Además, tienen ciclos de vida cortos y su mantenimiento y cultivo en el laboratorio son sencillos (Dean, 2008).

La toxicidad relativa de metales en poliquetos en orden decreciente es cobre, mercurio, zinc, arsénico, cromo y cadmio (Reish, 1988). Sin embargo, existen relativamente pocos estudios sobre el efecto del $\mathrm{Cd}$ en poliquetos. Reish \& Carr (1978) demostraron que el efecto del Cd sobre la supervivencia, reproducción, desarrollo y ciclos de vida de los poliquetos Ctenodrilus serratus (Schmidt, 1857) y Ophryotrocha diadema Åkesson, 1976, es mínimo en comparación con otros metales pesados como el cobre y mercurio. Los miembros de varias especies crípticas del complejo de Capitella capitata han sido objeto de estudio del efecto del $\mathrm{Cd}$ en varias respuestas fisiológicas. Los estudios pioneros demostraron que el $\mathrm{Cd}$ disuelto en agua es uno de los metales pesados menos dañinos para la supervivencia de Capitella spp. (Reish, Martin, Piltz, \& Word, 1976; Reish \& Le May, 1991). Estudios más recientes han demostrado la ausencia de efectos significativos del $\mathrm{Cd}$ incorporado en agua y sedimentos sobre las tasas de crecimiento, tasa de egestión y alometría de Capitella sp I (Selck et al., 1998; Selck, Decho, \& Forbes, 1999), mortalidad, producción de heces fecales y masa corporal de Capitella sp. I y Capitella sp. B (Méndez \& Baird, 2002) y mortalidad de juveniles de Capitella sp. Y (Méndez \& Green-Ruiz, 2005). Sin embargo, el estudio de Méndez \& Green-Ruiz (2006), demostró efectos del Cd sobre la mortalidad y metamorfosis de larvas de Capitella sp. Y.

A pesar de la relativamente baja toxicidad del Cd observada en algunos poliquetos, se espera que el elemento cadmio tenga efecto sobre la mortalidad de otras especies de poliquetos no estudiadas, demostrando así el impacto que este elemento puede tener en los ecosistemas naturales. Por otro lado, este estudio aportará nuevos conocimientos, debido a que los únicos trabajos de poliquetos en el Perú se restringen a estudios ecológicos y de diversidad, siendo el grupo menos estudiado en comparación con otros invertebrados bentónicos como moluscos, crustáceos y equinodermos, entre otros (Aguirre \& Canales, 2017; Vera, Tam, Vera, \& Pinto, 2001).

Por lo tanto, en este trabajo se pretende evaluar el efecto tóxico del Cd sobre la mortalidad de adultos de Polydora sp, el cual puede ser un buen candidato como especie prueba en futuros estudios ecotoxicológicos. 


\section{MATERIALES Y MÉTODOS}

El ensayo sobre el efecto del $\mathrm{Cd}$ en la mortalidad adultos de Polydora sp. se realizó durante la época de verano, entre diciembre del 2016 a marzo del 2017 en el Laboratorio de Ecotoxicología Acuática, Área Funcional de Investigaciones Marino Costeras (AFIMC) de la Dirección General de Investigaciones en Acuicultura (DGIA) del Instituto del Mar del Perú (IMARPE), Callao, Perú.

Individuos adultos de Polydora sp. (entre 5 y $5.3 \mathrm{~cm}$ de longitud, con 20 a 25 segmentos) se recolectaron en diciembre de 2016 en la zona intermareal del humedal Poza de la Arenilla, Malecón Pardo, distrito de La Punta, Callao

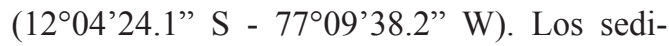
mentos en esta zona son predominantemente fangosos con contenidos de materia orgánica entre 2.53 y $22.8 \%$ (Velasco \& Solís, 2013). El estudio de Sánchez, Flores, \& Henostroza (2014) reveló concentraciones de $\mathrm{Cd}$ en sedimento entre 0.14 y $1.08 \mu \mathrm{g} / \mathrm{g}$ con valor medio de $0.68 \mu \mathrm{g} / \mathrm{g}$. En general el $100 \%$ de los valores registrados no superaron el Nivel Probable de Efecto estipulado en la Tabla de Protección Costera de EE UU $(4.21 \mu \mathrm{g} / \mathrm{g})$.

El sedimento superficial $(15 \mathrm{~cm}$ de profundidad) se recolectó con una espátula de aluminio y se tamizó con una malla de 500 $\mu \mathrm{m}$. El material biológico retenido en el tamiz se colocó en envases de plástico de $500 \mathrm{ml}$ con agua de mar para su traslado al laboratorio. La temperatura superficial del agua de mar fue de $32{ }^{\circ} \mathrm{C}$ y la salinidad de 29.6 ups, obtenidas con un equipo multiparamétrico portátil (Hach HQ40D). Estas mediciones fueron importantes para mantener condiciones similares en el laboratorio.

En el laboratorio, los especímenes fueron identificados utilizando un microscopio estereoscopio (Olympus SZ-61 ILST 1K36156). El género Polydora, junto con otros cinco géneros de la familia Spionidae, forma parte del complejo de especies de Polydora. El género Polydora se caracteriza por la presencia de setas modificadas en el setígero cinco (con espinas grandes de un solo tipo dispuestas en una sola hilera curveada, con setas de acompañamiento delgadas) y branquias a partir del setígero seis a 11. El setígero uno carece de notosetas. Los ganchos cubiertos neuropodiales son bidentados a partir de los setígeros siete al 14. El prostomio puede ser entero o con incisión y se extiende posteriormente como carúncula, la cual está rodeada de órganos nucales. La forma del pigidio es variable (Blake \& Woodwick, 1971; Delgado-Blas, 2008).

Para el mantenimiento de los especímenes en el laboratorio se acondicionaron mesas de enfriamiento reguladas a $20^{\circ} \mathrm{C}$. Se utilizaron: i) Dos vasos de precipitados de $1 \mathrm{~L}$ de capacidad y en cada uno se colocó una capa de 2 a 4 $\mathrm{cm}$ de sedimento experimental (Linke-Gamenick, Forbes, \& Méndez, 2000; Méndez, 2005, 2006a,b); ii) $500 \mathrm{ml}$ de agua de mar filtrada (< $1 \mu \mathrm{m})$ con salinidad de 30 ups; iii) se colocaron 150 individuos en cada vaso. El periodo de aclimatación a las condiciones de laboratorio duró dos semanas (Kenny, 1969). Para ello, los individuos adultos de Polydora sp se colocaron en envases de plástico de $1 \mathrm{~L}$ de capacidad con sedimento experimental y aireación constante durante dos semanas. Durante este periodo los organismos se alimentaron mediante la adición semanal de $0.5 \mathrm{~g}$ de alimento artificial seco que consistía en una mezcla, a partes iguales, de espinaca y comida para peces (Méndez, 2006a,b). Posteriormente, permanecieron tres días en agua de mar sin sustrato (Gopalakrishnan, Thilagam, \& Raja, 2007).

El sedimento experimental utilizado para la aclimatación de los especímenes se recolectó en el área de estudio, simultáneamente con las muestras biológicas, se secó en estufa a $80{ }^{\circ} \mathrm{C}$ durante 24 h (To-orn \& Paphavasit, 2017) y se tamizó a través de una malla de $250 \mu \mathrm{m}$ para utilizar la parte menor de esta fracción (Méndez, Lacorte, \& Barata, 2013), la cual corresponde a arena fina (Folk, 1965).

Diseño experimental: Se eligió el Cd con fines comparativos con otros poliquetos. Debido a la escasez de datos en la literatura sobre concentraciones subletales de $\mathrm{Cd}$ en solución para experimentos con poliquetos, se consideró 
la concentración mínima utilizada por Méndez \& Green-Ruiz (2006) con larvas de Capitella sp Y $(0.035 \mathrm{mg} / \mathrm{L})$ y, como concentración máxima, se consideró la $\mathrm{CL}_{50}$ a 96 horas de $75 \mathrm{mg} / \mathrm{L}$ registrada para el poliqueto Eurythoe complanata por Reish, Asato, \& LeMay (1989). Con el fin de determinar las concentraciones nominales de $\mathrm{Cd}$, se analizó un intervalo de cinco concentraciones de manera logarítmica, a fin de obtener un amplio intervalo de concentraciones, considerando estos valores máximo y mínimo. La ventaja de la escala logarítmica es que permite analizar los impactos en las tasas de mortalidad en función de diversos órdenes de magnitud en el cambio de la concentración. La concentración mayor $(75 \mathrm{mg} / \mathrm{L})$, se multiplicó por el factor de 0.15 (Yacomelo, 2014) y las multiplicaciones sucesivas por este factor permitieron calcular las cuatro concentraciones restantes. Así, las concentraciones nominales para el experimento fueron $75,11.25,1.69$, $0.25 ; 0.04 \mathrm{mg} / \mathrm{L}$ y un control.

La preparación de las cinco concentraciones se realizó por separado mediante la dilución de diferentes cantidades $\mathrm{CdCl}_{2}$ (tóxico de referencia considerado por Ingersoll et al. (1994)) en $3 \mathrm{~L}$ agua de mar filtrada $(<1 \mu \mathrm{m})$, con 30 a 32 ups, 20 a $21{ }^{\circ} \mathrm{C}$ y 8 a $9 \mathrm{mg} / \mathrm{L}$ de oxígeno disuelto. Para la preparación del tratamiento de $75 \mathrm{mg} / \mathrm{L}$, se diluyeron $225 \mathrm{mg}$ de $\mathrm{CdCl}_{2}$, para el segundo tratamiento se utilizaron $33.75 \mathrm{mg}$ de $\mathrm{CdCl}_{2}$, y así sucesivamente, para el resto de las concentraciones.

Durante el bioensayo, se colocaron diez individuos adultos en $1 \mathrm{~L}$ de solución de $\mathrm{Cd}$ con las diferentes concentraciones (y $1 \mathrm{~L}$ de agua de mar para los controles) y se realizaron tres réplicas por tratamiento. El experimento tuvo una duración de 72 horas, durante el cual no se colocó sedimento ni se administró alimento artificial a los especímenes para evitar la posible interacción con las soluciones de Cd. Los especímenes fueron contados cada 24 horas utilizando un microscopio estereoscópico, a fin de determinar su supervivencia.

Análisis Estadístico de los datos: El porcentaje de mortalidad para cada réplica se determinó de acuerdo con la expresión propuesta por Horng, Wang, \& Cheng (2009):

$$
\mathrm{S}=(\mathrm{nf} / \mathrm{ni}) * 100,
$$

Donde:

$\mathrm{nf}=$ número final de individuos vivos

$\mathrm{ni}=$ número inicial de individuos vivos

$\mathrm{S}=\%$ de supervivencia

Los datos de supervivencia fueron expresados como porcentaje de mortalidad, los cuales fueron utilizados para el cálculo de la la $\mathrm{CL}_{50}$, mediante el programa Probit (USEPA) en SPPS ver. 2.1, a fin de determinar la concentración letal media $\left(\mathrm{CL}_{50}\right)$.

Debido a que los datos no eran normales (prueba de Kolmogorov-Smirnov), se realizó una prueba de Kruskal-Wallis para observar si existen diferencias significativas entre los tratamientos. Posteriormente, se realizó la prueba de U-Man Whitney para detectar las diferencias entre pares de tratamientos. Para ambas pruebas se utilizó el programa SPPS ver. 2.1.

\section{RESULTADOS}

La tabla 1 muestra los resultados de mortalidad registrados durante los tres días que duró el bioensayo. La supervivencia disminuyó al aumentar la concentración de $\mathrm{Cd}$ y el efecto negativo del $\mathrm{Cd}$ fue mucho más evidente en los tratamientos 11.25 y $75 \mathrm{mg} / \mathrm{L}$. En ambos tratamientos, el efecto del tiempo de exposición fue más evidente a partir de las 24 horas experimentales, mientras que la mortalidad en los tratamientos con menores concentraciones de Cd fue evidente a partir del segundo día de exposición. Estos resultados fueron confirmados con la prueba de Kruskal-Wallis que mostró diferencias entre los tratamientos (Chi cuadrado $=10.536 ; \mathrm{gl}=4 ; \mathrm{p}=0.032$ ) y la prueba de U-Man Whitney en la que los tratamientos 11.25 y $75 \mathrm{mg} / \mathrm{L}$ presentaron diferencias con los tres tratamientos restantes. El control presentó diferencias con todos los tratamientos de Cd. $\mathrm{La} \mathrm{CL}_{50}$ de Cd durante $72 \mathrm{~h}$ fue de 2.59 
TABLA 1

Mortalidad (\%) registrada durante el bioensayo sobre adultos de Polydora.

TABLE 1

Mortality (\%) registered during the bioassay on adults of Polydora.

\begin{tabular}{cccccccc} 
Tiempo & Réplicas & Control & $0.04 \mathrm{mg} / \mathrm{L}$ & $0.25 \mathrm{mg} / \mathrm{L}$ & $1.6 \mathrm{mg} / \mathrm{L}$ & $11.25 \mathrm{mg} / \mathrm{L}$ & $75 \mathrm{mg} / \mathrm{L}$ \\
\hline \multirow{5}{*}{4 horas horas } & R1 & 0 & 0 & 0 & 0 & 0 & 0 \\
& R2 & 0 & 0 & 0 & 0 & 0 & 0 \\
& R3 & 0 & 0 & 0 & 0 & 0 & 0 \\
& R1 & 0 & 0 & 0 & 0 & 100 & 100 \\
& R2 horas & 0 & 20 & 0 & 0 & 100 & 100 \\
& R3 & 0 & 0 & 0 & 0 & 100 & 100 \\
& R1 & 0 & 10 & 10 & 40 & 100 & 100 \\
& R2 & 0 & 30 & 100 & 0 & 100 & 100 \\
\hline
\end{tabular}

$\mathrm{mg} / \mathrm{L}$, con límite superior de y $16.39 \mathrm{mg} / \mathrm{L}$ y límite inferior de $1.37 \mathrm{mg} / \mathrm{L}$ (Fig. 1).

La mortalidad fue comprobada al estimular a los organismos con una varilla de vidrio y observar la carencia de movimiento Los síntomas de mortalidad fueron más evidentes en los tratamientos 11.25 y $75 \mathrm{mg} / \mathrm{L}$, ya que los organismos disminuyeron su movilidad paulatinamente hasta la muerte Asimismo, tendían a agruparse y a formar tubos de sedimento. La mayoría de los especímenes de estos tratamientos cambiaron su coloración de rojiza a verde y algunos organismos presentaron pequeñas carnosidades en los setígeros.

\section{DISCUSIÓN}

En la actualidad únicamente existen dos estudios de impacto ambiental en el área de estudio. Troll (2000), realizó una evaluación y ordenamiento ambiental para el establecimiento de un área protegida en la Poza de La

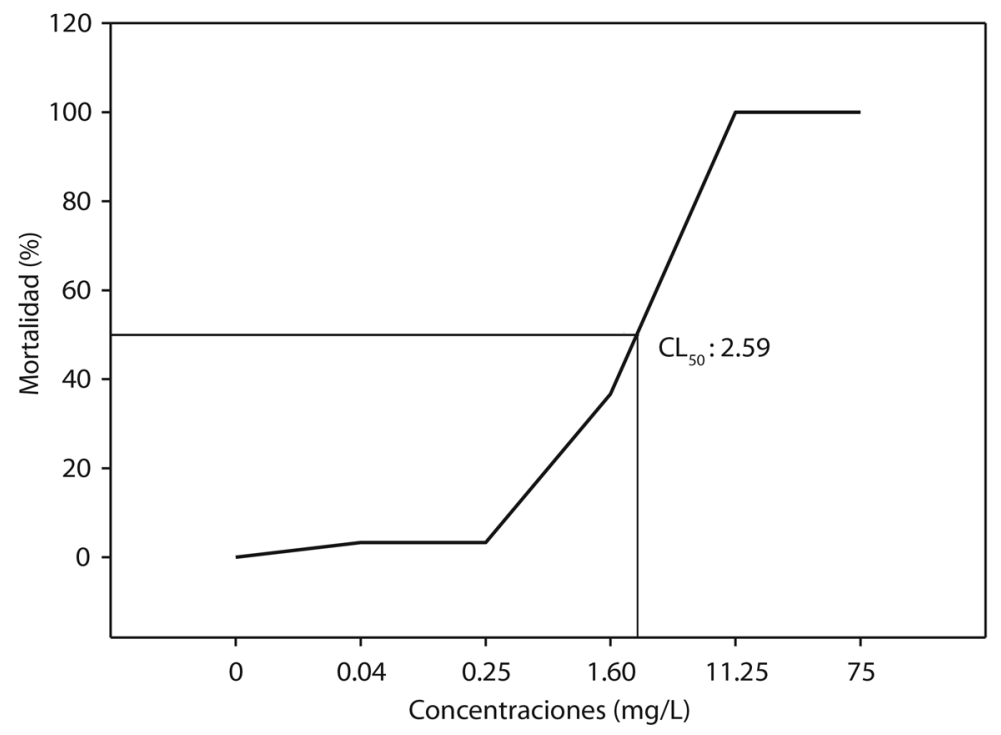

Fig. 1. Concentración letal media $\left(\mathrm{CL}_{50}\right)$ a $72 \mathrm{~h}$ durante el bioensayo sobre adultos de Polydora.

Fig. 1. Median lethal dose $\left(\mathrm{LC}_{50}\right)$ at $72 \mathrm{~h}$ during the bioassay on adults of Polydora. 
Arenilla, mediante cuatro puntos de monitoreo de agua. Estos resultados fueron comparados con los estándares de calidad ambiental correspondientes y concluyó que las concentraciones de $\mathrm{Cd}$ en todos los puntos fueron inferiores a $0.001 \mathrm{mg} / \mathrm{L}$, lo que indica que estos valores se encuentran muy por debajo de la cuantificación del método del laboratorio Estos resultados coinciden con los registros de Sánchez et al. (2014) en el estudio de calidad ambiental del humedal Poza La Arenilla-Callao en 2008, en el cual los valores de $\mathrm{Cd}$ estuvieron por debajo del límite de detección del equipo, lo que explica la supervivencia de Polydora sp. en ese ambiente.

Dada la escasez de estudios ecotoxicológicos con poliquetos, los datos de referencia de concentraciones de $\mathrm{Cd}$ experimentales son escasos, por lo que nuestras concentraciones nominales fueron calculadas en base a las escalas de otras especies de poliquetos registradas por Reish et al (1989) y Méndez \& Green-Ruiz (2006). Los valores de mortalidad mostraron que el intervalo de concentraciones nominales elegido en este estudio preliminar superó los valores de concentraciones subletales, ya que en las concentraciones 11.25 y 75 $\mathrm{mg} / \mathrm{L}$ murieron todos los organismos durante las primeras 24 horas. Por el contrario, los resultados de mortalidad en los tratamientos menores a $0.25 \mathrm{mg} / \mathrm{L}$ están en el mismo orden de magnitud que las concentraciones utilizadas por Selck et al $(1998,1999)$ y por Méndez \& Green-Ruiz $(2005,2006)$ con Capitella sp. I y Capitella sp. Y, respectivamente. Estos resultados sugieren que para futuros estudios en los que se analicen otras respuestas de este organismo se deberían utilizar concentraciones subletales de $\mathrm{Cd}$ menores a $1.6 \mathrm{mg} / \mathrm{L}$, ya que en este tratamiento se observó mortalidad después de dos días. Idealmente, deberían realizarse más estudios preliminares detallados utilizando concentraciones bajas entre 0.04 y $1.6 \mathrm{mg} / \mathrm{L}$, de preferencia con individuos identificados a nivel de especie (ya que las respuestas podrían verse enmascaradas a niveles taxonómicos superiores).
La comparación de la $\mathrm{CL}_{50}$ con otros poliquetos expuestos a $\mathrm{Cd}$ en solución (procedentes de cultivos realizados en Los Ángeles, EE UU) muestra que el valor obtenido con Polydora sp. durante 72 horas $(2.59 \mathrm{mg} / \mathrm{L})$ está en el mismo orden de magnitud que los valores registrados para adultos de Capitella capitata (Fabricius, 1780) $(2.8 \mathrm{mg} / \mathrm{L})$ y Pectinaria californiensis Hartman, 1941 (2.6 mg/L) (Reish \& Le May, 1991) y de Neosabellaria clandestinus (Menon \& Sareen, 1966) (2.8 mg/L; como Sabellaria clandestinus en Mohan, Menon, \& Gupta, 1984) en sus experimentos de 96 horas de exposición. Estos valores resultaron menores que los de los poliquetos Neanthes arenaceodentata $(14.1 \mathrm{mg} / \mathrm{L})$ Nereis grubei $(4.7 \mathrm{mg} / \mathrm{L})$ y Ophryotrocha labronica Bacci \& La Greca, 1961 (3.7 mg/L), de los mismos autores, así como los de $C$ serratus y $O$ diadema $(10 \mathrm{mg} / \mathrm{L})$ de Reish \& Carr (1978). Estas comparaciones indican que los adultos de Polydora sp. presentan mayor sensibilidad al Cd que otras especies, ya que existe mortalidad en menores concentraciones de $\mathrm{Cd}$, lo que la señala como una buena especie de prueba para futuros estudios de ecotoxicología. Por el contrario, la $\mathrm{CL}_{50}$ de Polydora sp. resultó mayor que la obtenida para larvas trocóforas de $C$. capitata durante 96 horas $(0.22 \mathrm{mg} / \mathrm{L}$; Reish et al., 1976) y para larvas metatrocóforas de Capitella sp. Y procedentes de Mazatlán, México $(0.035 \mathrm{mg} / \mathrm{L}$, Méndez \& Green-Ruiz, 2006) en un periodo de 10 días. Esto sugiere que dentro del grupo de los poliquetos, el Cd tiene un impacto mayor en estados larvarios, ya que la mortalidad ocurre a menores concentraciones que en los adultos.

De acuerdo con Delgado-Blas (2009), los miembros de la familia Spionidae, en el medio natural, tienen la capacidad de cambiar su patrón de consumidores de depósito a suspensívoros. Esto podría explicar el éxito del experimento, ya que los controles sobrevivieron sin sedimento durante los tres días experimentales, lo que indica que probablemente cambiaron el patrón de alimentación para sobrevivir, mientras que los organismos expuestos recibieron el efect $f$ o adverso del $\mathrm{Cd}$ provocándoles la muerte. Estos resultados concuerdan con las 
características establecidas por Reish (1980) sobre el valor de los poliquetos en estudios de laboratorio (relativamente fácil recolección, manipulación, cultivo y transporte y ciclos de vida relativamente cortos). Nuestros resultados apoyan la sugerencia de utilizar Polydora spp. como otra especie prueba dentro del grupo de los poliquetos, en experimentos de ecotoxicología en ecosistemas marino-costeros en Perú o en otras localidades. Además, se podrán sentar las bases para realizar estudios ecotoxicológicos y fisiológicos con otros géneros de poliquetos frente a otros tóxicos, así como estudios de bioacumulación y biomarcadores enzimáticos o moleculares incentivando mayor interés de estudio sobre la clase Polychaeta.

Los estudios ecotoxicológicos son importantes, ya que permiten identificar problemas ambientales, establecer controles de contaminación, establecer límites, identificar apropiadamente acciones correctivas y monitorear los efectos de efluentes contaminados (Wall \& Hanmer, 1987; Fent, 2003). La presente investigación preliminar constituye el primer estudio ecotoxicológico en Perú con el grupo Polychaeta en condiciones de laboratorio. Este estudio puede contribuir a sentar las bases para futuros estudios de ecotoxicología, monitoreo biológico y calidad ambiental utilizando el complejo de especies de Polydora, así como otros géneros de la familia Spionidae.

Declaración de ética: los autores declaran que todos están de acuerdo con esta publicación y que han hecho aportes que justifican su autoría; que no hay conflicto de interés de ningún tipo; y que han cumplido con todos los requisitos y procedimientos éticos y legales pertinentes. Todas las fuentes de financiamiento se detallan plena y claramente en la sección de agradecimientos. El respectivo documento legal firmado se encuentra en los archivos de la revista.

\section{AGRADECIMIENTOS}

Se agradece a Christian Paredes, Laboratorio de Ecotoxicología Acuática, del Instituto del Mar del Perú (IMARPE).

\section{RESUMEN}

Introducción: diversos estudios han demostrado el efecto del Cd sobre la mortalidad y otras respuestas fisiológicas de diversas especies de poliquetos. Objetivo: evaluar el efecto tóxico del $\mathrm{Cd}$ en la mortalidad de adultos de Polydora sp. como especie prueba para estudios ecotoxicológicos. Métodos: los especímenes fueron recolectados en el humedal costero Poza de la Arenilla, Callao, Peru. Los organismos fueron aclimatados durante dos semanas con agua de mar y sedimento y posteriormente, tres días en agua de mar, con $30 \%$ de salinidad y temperatura entre 20 a $22{ }^{\circ} \mathrm{C}$. Los organismos se expusieron a las concentraciones nominales $75,11.25,1.69,0.25 ; 0.04 \mathrm{mg} / \mathrm{L}$ de $\mathrm{Cd}$ y un control. Se analizaron tres réplicas de diez individuos por tratamiento durante 72 horas para registrar mortalidad. Se aplicaron las pruebas de Kruskal-Wallis y de U-Man Whitney para detectar las diferencias significativas entre tratamientos. Resultados: el efecto adverso del Cd sobre la mortalidad fue mucho más evidente en los tratamientos de 11.25 y $75 \mathrm{mg} / \mathrm{L}$ a partir de las primeras 24 horas de exposición. La concentración letal media de $\mathrm{Cd}$ a $72 \mathrm{~h}$ fue de 2.59 mg/L. Conclusiones: la $\mathrm{CL}_{50}$ está en el mismo orden de magnitud de otras especies de poliquetos. Este es el primer estudio ecotoxicológico en Perú con poliquetos en condiciones de laboratorio. Se sugiere la utilización de Polydora sp. como especie prueba en experimentos de ecotoxicología, utilizando concentraciones nominales subletales de $\mathrm{Cd}$ que no superen los $1.6 \mathrm{mg} / \mathrm{L}$ para la observación de varias respuestas fisiológicas.

Palabras clave: Ecotoxicología, poliquetos, metales pesados, $\mathrm{CL}_{50}$, bioensayos, especie prueba, humedal costero Poza de la Arenilla.

\section{REFERENCIAS}

Aguirre, L., \& Canales, R. (2017). Poliquetos de Perú: estado actual y perspectivas para la investigación. En O. Díaz-Díaz, D. Bone, C. T. Rodríguez \& V. H. Delgado-Blas (Eds.). Poliquetos de Sudamérica (pp. 101-114). Cumaná, Venezuela: Volumen especial del Boletín del Instituto Oceanográfico de Venezuela.

Ajao, E. A., \& Fagade, S. O. (1990). A Study of the sediments and communities in Lagos Lagoon, Nigeria. Oil \& Chemical Pollution, 7, 85-117.

Ankley, G. T., Thomas, N. A., DiToro, D. M., Hansen, D. J., Mahony, J. D., Berry, W. J., ... Zarba, C. S. (1994). Assesing potential bioavailability of metals in sediments: a proposed approach. Environmental Management, 18, 331-337.

Blake J. A., \& Woodwick, K. H. (1971). New species of Polydora (Polychaeta: Spionidae) from the Coast of California. Bulletin of the South California Academy of Sciences, 70(2), 72-79. 
Correa, D., Tam, J., Pasapera, J., Saavedra, M. \& Ingunza, A. (2008). Modelado de la circulación marina y descargas hipotéticas en la bahía del Callao, Perú. Informe IMARPE, 35(3), 181-192.

Dean, H. K. (2008). The use of polychaetes (Annelida) as indicator species of marine pollution: a review. Revista de Biologia Tropical, 56(4), 11-38.

Delgado-Blas, V. H. (2008). Polydora and related genera (Polychaeta: Spionidae) from the Grand Caribbean region. Journal of Natural History, 42(1-2), 1-19.

Delgado-Blas, V. H.. (2009). Spionidae Grube, 1850. En J. A. de León-González, R. Bastida-Zavala, L. F. Carrera-Parra, P. Hernández-Alcántara, S. I. SalazarVallejo, V. Solís-Weiss (Eds.). Poliquetos (Annelida: Polychaeta) de México y América Tropical (pp. 586614). Monterrey, México: Universidad Autónoma de Nuevo León.

Fauchald, K., \& Jumars, P. A. (1979). The diet of worms: a study of polychaete feeding guilds. Oceanography and Marine Biology. An Annual Review, 17, 193-284.

Fent, K. (2003). Ecotoxicological problems associated with contaminated sites. Toxicological Letters, 140-141, $353-365$.

Fernández-Rodríguez, V., \& Londoño-Mesa, M. (2015). Poliquetos (Annelida: Polychaeta) como indicadores biológicos de contaminación marina: casos en Colombia. Gestión y Ambiente, 18, 189-204.

Folk, R.L. (1965). Petrology of Sedimentary Rocks. Austin, USA: Hemphills Publications Company.

Förstner, U., \& Wittmann, G. T. W. (1981). Metal pollution in the aquatic environment. Second revision. Berlin, Germany: Springer-Verlag.

Fu-hong, S., \& Qi-xing, Z. (2008). Oxidative stress biomarkers of the polychaete Nereis diversicolor exposed to cadmium and petroleum hydrocarbons. Ecotoxicology and Environmental Safety, 70, 106-114.

Gopalakrishnan, S., Thilagam, H., \& Raja, P. V. (2007). Toxicity of heavy metals on embryogenesis and larvae of the marine sedentary polychaete Hydroides elegans. Archives of Environmental and Contamination Toxicology, 52, 171-178.

Horng, C. Y., Wang, S. L, \& Cheng, I. J. (2009). Effects of sediment-bound $\mathrm{Cd}, \mathrm{Pb}$, and $\mathrm{Ni}$ on the growth, feeding, and survival of Capitella sp. I. Journal of Experimental Marine Biology and Ecology, 371, 68-76.

Ingersoll, C. G., Ankley, G., Burton Jr, G. A., Dwyer, F. J., Hoke, R., Norberg-King, T., ... Winger, P. V. (1994). Methods for Measuring the Toxicity and Bioaccumulation of Sediment-Associated Contaminants With Freshwater Invertebrates. Washington D. C., USA: US Environmental Protection Agency.
Jenkins, K. D., \& Sanders, B. M. (1986). Relationships between Free Cadmium Ion Activity in Seawater, Cadmium Accumulation and Subcellular Distribution and Growth in Polychaetes. Environmental Health Perspectives, 65, 205-210.

Kenny, R. (1969). Temperature tolerance of the polychaete worms Diopatra cuprea and Clymenella torquata. Marine Biology, 4, 219-223.

Linke-Gamenick, I., Forbes, V. E., \& Méndez, N. (2000). Effects of chronic fluoranthene exposure on sibling species of Capitella with different development modes. Marine Ecology Progress Series, 203, 191-203.

Méndez, N., \& Baird, D. J. (2002). Effects of cadmium on sediment processing in members of the Capitella species-complex. Environmental Pollution, 120, 299-305.

Méndez, N. (2005). Effects of teflubenzuron on larvae and juveniles of the polychaete Capitella sp B from Barcelona, Spain. Water, Air, and Soil Pollution, 160, 259-269.

Méndez, N. (2006a). Effects of teflubenzuron on sediment processing by members of the Capitella speciescomplex. Environmental Pollution, 139, 118-124.

Méndez, N. (2006b). Life cycle of Capitella sp. Y (Polychaeta: Capitellidae) from Estero del Yugo, Mazatlan, Mexico. Journal of the Marine Biology Association of the United Kingdom, 86, 263-269.

Méndez, N., \& Green-Ruiz, C. (2005). Preliminary observations of cadmium and copper effects on juveniles of the polychaete Capitella sp Y from Estero del Yugo, Mazatlan, Mexico. Revista Chilena de Historia Natural, 78, 701-710.

Méndez, N., \& Green-Ruiz, C. (2006). Cadmium and copper effects on larval development and mortality of the polychaete Capitella sp Y from Estero del Yugo, Mazatlan, Mexico. Water, Air, and Soil Pollution, 171, 291-299.

Méndez, N., Lacorte, S., \& Barata, C. (2013). Effects of the pharmaceutical fluoxetine in spiked-sediments on feeding activity and growth of the polychaete Capitella teleta. Marine Environmental Research, 89, 76-82.

Mohan, C. V., Menon, N. R., \& Gupta, T. R. C. (1984). Acute toxicity of cadmium to 6 intertidal invertebrates. Fishery Technology, 21, 1-5.

Nürnberg, H. W. (1977) Potentialities and applications of advanced polarographic and voltammetric methods in environmental research and surveillance of toxic metals. Electrochimica Acta, 22, 935-949.

Reish, J. D. (1980). Use of Polychaetous Annelids as test organisms for marine bioassay experiments. En A. 
L. Jr. Buikema \& J. Cairns Jr. (Eds). Aquatic Invertebrate bioassays (pp. 140-154). Philadelphia, USA: ASTM STP 715. American Society for Testing and Materials.

Reish, J. D. (1988) The use of toxicity testing in marine environmental research. En D. F. Soule, \& G. S. Kleppel (Eds.), Marine organisms as indicators (pp. 231-245). New York, USA: Springer-Verlag.

Reish, D. J., Asato, S. L., \& LeMay, J. A. (1989). The effects of cadmium and DDT on the survival and regeneration in the amphinomidae polychaete Eurythoe complanata. En VII Simposio Internacional de Biología Marina (pp. 107-111). La Paz, México: Universidad Autónoma de Baja California Sur.

Reish, D. J., \& Carr, R. S. (1978). The effect of heavy metals on the survival, reproduction, development, and life cycles of two species of polychaetous annelids. Marine Pollution Bulletin, 9, 24-27.

Reish, J. D., \& LeMay, J. A. (1991). Toxicity and bioconcentration of metals and organic compounds by polychaeta. Ophelia Supplement, 5, 653-660.

Reish, D. J., Martin, J. M., Piltz, F. M., \& Word, J. Q (1976). The effect of heavy metals on laboratory populations of two polychaetes with comparisons to the water quality conditions and standards in Southern California marine waters. Water Research, 10, 299-302.

Sánchez, R. G., Orozco, R., \& Guzmán, M. (2002). Bahía del Callao, Perú. Evaluación ambiental en marzo 2002. Informe Instituto del Mar del Perú, 35, 27-41.

Sánchez, R. G., Flores, V., \& Henostroza, A. (2014). Calidad Ambiental del Humedal Poza La Arenilla-Callao. Informe Instituto del Mar del Perú, 41(1-4), 202-214.

Selck, H., Forbes, V. E., \& Forbes, T. L. (1998). Toxicity and toxicokinetics of cadmium in Capitella sp. I: relative importance of water and sediment as routes of cadmium uptake. Marine Ecology Progress Series, 164, 167-178.
Selck, H., Decho, A. W., \& Forbes, V. E. (1999). Effects of chronic metal exposure and sediment organic matter on digestive absorption efficiency of cadmium by the deposit-feeding polychaete Capitella species I. Environmental Toxicology and Chemistry, 18(6), 1289-1297.

To-orn, N., \& Paphavasit, N. (2017). Bioremediation of organically enriched sediment under green mussel rafts using Spionids Genus Prionospio (Polychaeta: Spionidae). EnvironmentAsia, 10(1), 17-24.

Tomassetti, P., \& Porrello, S. (2005). Polychaetes as indicators of marine fish farm organic enrichment. Aquaculture International, 13, 109-128.

Tovar-Hernández, M. A., Salazar-Silva, P., de León-González, J. A., Carrera-Parra, L. F., \& Salazar-Vallejo, S. I. (2014). Biodiversdiad de Polychaeta (Annelida) en México. Revista Mexicana de Biodiversidad, 85, 190-196.

Troll, J. L. (2000). Evaluación y ordenamiento ambiental para el establecimiento de un área protegida en la poza de La Arenilla, La Punta, Callao (Tesis de Licenciatura). Perú: Universidad Particular Ricardo Palma.

Velazco, C. F. J., \& Solís, A. J. (2013). Caracterización de los sedimentos de la Poza La Arenilla-Callao, marzo 2004. Informe Técnico del Instituto del Mar del Perú, 40 (3-4), 146-149.

Vera, G., Tam, J., Vera, V., \& Pinto, E. (2001). Pruebas ecotoxicológicas con Cadmio y Cromo usando postlarvas de Pejerrey Odontesthes (Austromenidia) regia regia Hildebrand. Revista Peruana de Biología, 8(2), 125-135.

Wall, T. M., \& Hanmer, R. W. (1987). Biological Testing to Control Toxic Water Pollutants. Water Environmental research, 59(1), 7-12.

Yacomelo, H. M. J. (2014). Riesgo toxicológico en personas expuestas a suelos y vegetales, con posibles concentraciones de metales pesados, en el Sur del Atlántico, Colombia (Tesis de Maestría). Medellín, Colombia: Universidad de Colombia. 